\title{
The first simultaneous pancreas kidney transplantion in Sri Lanka
}

\author{
J. Arudchelvam, A. Marasinghe, R. Dassanayake, S. Anver, P. Rajakrishna \\ Teaching Hospital Anuradhapura, Sri Lanka
}

Keywords: Simultaneous pancreas kidney transplant; renal transplantation; pancreas transplantation; renal failure

\section{Introduction}

Diabetes Mellitus (DM) is a common disease in Sri Lanka with a prevalence of $10.3 \%$ in individuals of more than 20 years of age [1]. Although, most of these patients can be managed with oral hypoglycaemics and insulin (both type 1 and 2) in about $15 \%$ of patients this becomes difficult [2]. When renal failure develops in these patients Simultaneous Pancreatic Kidney Transplantation (SPK) becomes an option. Such transplantation has not been done in Sri Lanka before. The aim of this article is to report the first successful SPK in Sri Lanka which is also the first dual organ transplantation in this country.

\section{Case report}

A 47 year old male patient with type 2 DM and End Stage Renal Failure (ESRF) was prepared for SPK. his blood sugar control was erratic despite being on high doses of insulin $(30 \mathrm{u}$ mane and $40 \mathrm{u}$ nocte of mixtard (30/70) insulin) with usual blood sugar levels between $250 \mathrm{mg} / \mathrm{dl}$ to $300 \mathrm{mg} / \mathrm{dl}$ and 3 admissions with hypoglycaemia in last 3 months. The patient was diagnosed of having renal failure for last 6 months and was on twice a week haemodialysis for 3 months. He was on a deceased donor waiting list for renal transplantation at The Teaching Hospital Anuradhapura, Sri Lanka.

A 41 year old brain dead compatible donor became available. Pancreas was retrieved with coeliac axis, superior mesenteric artery, portal vein and duodenal stump. The left kidney was also harvested for transplantation. HTK (Histidine Ketoglutarate Tryptophan) solution was used for cold perfusion and preservation. Iliac artery "Y" graft was taken from the donor. The coeliac axis and superior mesenteric stumps were connected using the "Y" graft.

A Midline laparotomy was made on recipient. Pancreatic vessels were anastomosed to external iliac vessels on the right

Correspondence: J. Arudchelvam

E-mail: joelaru@yahoo.com

DOI: http://10.4038/sljs.v34i4.8323 side. Duodenal stump was anastomosed to terminal ileum forming an enteric drainage (Figure 1). Renal vessels were anastomosed to left external iliac vessels and ureter was anastomosed to bladder over $5 \mathrm{~F}$ double "J" stent.

The blood sugar level became normal from post op day 2 and he did not require insulin after that. The patient developed a retro-pancreatic haematoma which was evacuated on day 5. He was discharged home on day 15 with Tacrolimus, Mycophenolate Mofetil (MMF) and prednisolone as antirejection medications.

At present 2 months after SPK patient is well with the fasting blood sugar range of 97 to $130 \mathrm{mg}$ / $\mathrm{dl}$ and serum creatinine of 84 to $130 \mu \mathrm{mol} / 1$.

\section{Discussion and conclusions}

Pancreatic transplant was first performed in 1966 (3). Since then the surgical techniques and immunosuppression have evolved gradually. Because SPK improves the quality of life (4), reduces the progression and complications of DM (4) as well as improving overall survival more than those patients undergoing renal transplantation alone (5), at present SPK remains an established treatment for patients with uncontrolled DM and end stage diabetic nephropathy. DM is found in approximately $10.3 \%$ of population in Sri Lanka (1) and 10 to $20 \%$ of diabetic patients develop ESRF. Initially SPK was performed in patients with type $1 \mathrm{DM}$, but current studies have found similar benefits and patient and graft survival in patients with both types 1 and 2 DM (6). Therefore SPK should be considered in selected patients with DM and ESRF. Also establishing a countrywide deceased donor programme will facilitate achieving the above goal.

\section{References}

1. Katulanda P, Constantine GR, Mahesh JG, Sheriff R, Seneviratne RDA, et al. Prevalence and projections of diabetes and prediabetes in adults in Sri Lanka-Sri Lanka Diabetes, Cardiovascular Study (SLDCS). Diabetes UK 2008; 25: 1062-69 https://doi.org/10.1111/j.1464-5491.2008.02523.x

2. Somasundaram, G. N., S. D. Jayaratne, and N. P. Somasundaram. "Brittle diabetes." Journal of the Ceylon College of Physicians 37 (2004): 39-43

3. Kelly WD, Lillehei RC, Merkel FK, et al. Allotransplantation of 
the pancreas and duodenum along with the kidney in diabetic nephropathy. Surgery. 1967; 61:827-37

4. Milde FK, Hart LK, Zehr PS.: Pancreatic transplantation. Impact on the quality of life of diabetic renal transplant recipients. Diabetes Care 18: 93-95, 1995 https://doi.org/10.2337/diacare.18.1.93

5. 18. McCullough KP, Keith DS, Meyer KH, et al. Kidney and pancreas transplantation in the United States, 1998-2007:
Access for patients with diabetes and end-stage renal disease. Am J Transplant. 2009; 9:894-906.

https://doi.org/10.1111/j.1600-6143.2009.02566.x

6. Sampaio MS, Kuo H-T, Bunnapradist S. Outcomes of Simultaneous Pancreas-Kidney Transplantation in Type 2 Diabetic Recipients. Clinical Journal of the American Society of Nephrology : CJASN. 2011; 6(5):1198-1206. https://doi.org/10.2215/CJN.06860810

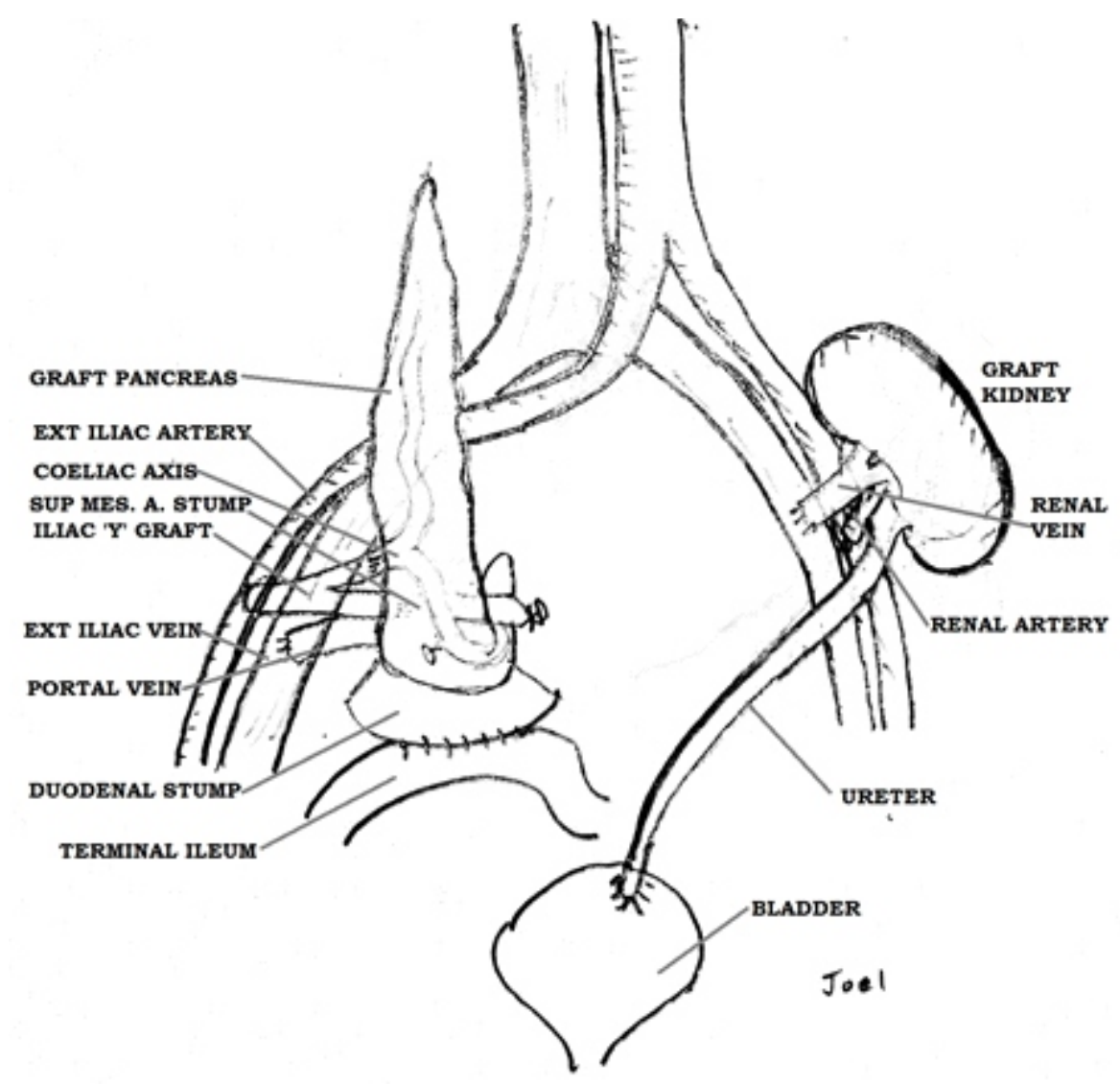

Figure 1. SPK Surgical Technique

\section{Key Points:}

- Simultaneous Pancreas and Kidney transplantation should be considered as an option in patients with renal failure and Diabetes Mellitus, especially when there are other complications of Diabetes Mellitus are developing and the blood sugar control becomes difficult.

- Adeceased donor program should be established in Sri Lanka to make such transplants possible. 